\title{
Cold Hypersensitivity in the Hands and Feet May Be Associated with Functional Dyspepsia: Results of a Multicenter Survey Study
}

\author{
Kwang-Ho Bae, 1 Ju Ah Lee, ${ }^{2}$ Ki-Hyun Park, ${ }^{1}$ Jong-Hyang Yoo, \\ Youngseop Lee, ${ }^{1}$ and Siwoo Lee ${ }^{1}$ \\ ${ }^{1}$ Mibyeong Research Center, Korea Institute of Oriental Medicine, 1672 Yuseongdae-ro, Yuseong-gu, Daejeon 305-811, Republic of Korea \\ ${ }^{2}$ KM Fundamental Research Division, Korea Institute of Oriental Medicine, 1672 Yuseongdae-ro, Yuseong-gu, \\ Daejeon 305-811, Republic of Korea
}

Correspondence should be addressed to Youngseop Lee; rheey119@kiom.re.kr and Siwoo Lee; ifree72@gmail.com

Received 25 September 2015; Revised 27 December 2015; Accepted 17 February 2016

Academic Editor: Sunoh Kwon

Copyright (c) 2016 Kwang-Ho Bae et al. This is an open access article distributed under the Creative Commons Attribution License, which permits unrestricted use, distribution, and reproduction in any medium, provided the original work is properly cited.

\begin{abstract}
Aim. To investigate whether dyspepsia symptoms differ depending on the presence or absence of cold hypersensitivity in the hands and feet (CHHF). Methods. In all, 6044 patients were recruited and provided with a questionnaire about CHHF and dyspepsia. Based on their responses, subjects were divided into a CHHF group (persons who noted cold sensations; $n=1209$ ) and a nonCHHF group (persons who noted warm or intermediate sensations; $n=1744$ ). The groups were compared in terms of their usual digestion status, using chi-square tests and logistic regression analyses to calculate the propensity score and odds ratios (ORs). We analyzed the participants' responses to questions on dyspepsia symptoms. Results. After matching, chi-square tests indicated that the CHHF group had higher frequencies of the following symptoms: bad digestion, poor appetite, discomfort in the upper abdomen, motion sickness, epigastric burning, postprandial fullness, nausea, and bloating. Additionally, CHHF was associated with an increased OR for dyspepsia (bad digestion, vomiting, motion sickness, epigastric burning, postprandial fullness, nausea, epigastric pain, and bloating) compared with the non-CHHF group. Conclusion. This study confirmed that CHHF patients have elevated frequencies of most dyspepsia symptoms.
\end{abstract}

\section{Introduction}

In traditional Korean medicine, pattern identification is important to both understanding the patient's status and writing a prescription. There are several methods of pattern identification, including 8-principle pattern identification, constitutional pattern identification, and visceral pattern identification $[1,2]$. The 8-principle system includes the following factors: yin and yang, exterior and interior, cold and heat, and deficiency and excess. Cold and heat is an important factor for revealing the patient's status. In Korean medicine, cold and heat do not refer to the patient's body temperature alone but instead are an inclusive concept that incorporates the patient's subjective feeling of warmth and chill, as well as fevers diagnosed by a doctor (4 examinations). Cold and heat is a phenomenon that appears when functional activities decline or increase due to diseases or constitution.
Because cold and heat pattern identification is diagnosed based on both the patient's symptoms and 4 examinations by a doctor of Korean medicine, objective evidence is necessarily insufficient for diagnosis. Further, evidence on the effects of cold and heat status on the body also remains uncertain. To resolve these issues, recent studies have attempted to provide objective standards for cold and heat, including the development of a cold pattern questionnaire by Ryu et al. [3] and a study in which Song et al. [4] investigated the dependence of Korean medicine prescriptions on cold and heat disposition in knee osteoarthritis. However, studies of cold and heat and the effects of their status on the body have been performed rarely to date. The rarity of such studies may be explained by the absence of verified diagnostic tools for cold and heat pattern identification; there is considerable uncertainty when conducting a study of cold and heat 
with relatively broad inclusion criteria and an open-ended definition of the pattern.

Therefore, we decided to conduct the present study using a narrower range of inclusion criteria and a more specific scope of research. First, rather than investigating the dual states of cold and heat, we limited our investigation to differences between cold and noncold individuals. Second, rather than recruiting subjects based on various symptoms of coldness, we specifically analyzed cold hypersensitivity in the hands and feet (CHHF), which is a representative cold symptom. CHHF is relatively common symptom in Korea and is more frequently observed in women than in men [5]. Patients with CHHF feel coldness both in cold places and at temperatures that are relatively warm. The prevalence rate of $\mathrm{CHHF}$ is somewhat uncertain because there is insufficient data to obtain an accurate estimate; however, $38.7 \%$ of women complained of coldness in a study by Kondo and Okamura [6]. Third, to investigate the effects of cold on body function, we specifically investigated differences in digestive function between the CHHF and non-CHHF groups via functional dyspepsia symptoms. Our decision to investigate digestive function was based on the relatively high prevalence rate of functional dyspepsia (8-30\%) [7] and the "spleen and 4 extremities" theory of the Huangdi Neijing [2], which states that the limbs are connected to the spleen. This theory implies that digestive function affects the 4 limb extremities, meaning that the limbs are healthy when the digestive function is healthy and diseased when the digestive function is poor.

Previous studies have also reported that cold hypersensitivity and dyspepsia are correlated $[8,9]$. However, those studies targeted limited subjects such as women or patients of a specific age group or involved an insufficient sample size. Thus, additional studies are necessary to clearly demonstrate this relationship. With these considerations in mind, we hypothesized that individuals with CHHF would have a poorer digestion status than those without CHHF. Accordingly, we investigated differences in digestion status among persons with and without CHHF by analyzing responses to a questionnaire.

\section{Methods}

2.1. Data Collection. This cross-sectional study was conducted between November 2006 and August 2014. All of the questionnaire data, including CHHF and dyspepsia status, were compiled from the Korean Medicine Data Center (KDC) of the Korea Institute of Oriental Medicine (KIOM) [10]. Using this resource, we collected questionnaire data on 6044 adults (19 years old or older) who were admitted to 13 traditional Korean medicine hospitals and 11 traditional Korean medicine clinics. To isolate our analysis from any effects of organic dyspepsia, we excluded data on patients diagnosed with chronic gastritis, gastroduodenal ulcers, esophagitis, fatty liver, hepatitis, or digestive tract tumors. After applying these exclusions, 3558 individuals remained. Among them, patients were excluded who showed unclear symptoms for classification into the CHHF group or nonCHHF group. The remaining 2953 individuals were selected as the final study subjects, including 1209 persons in the
CHHF group and 1744 persons in the non-CHHF group (Figure 1). This study was approved by the Institutional Review Board of KIOM (I-0910/02-001).

2.2. Cold Hypersensitivity in the Hands and Feet. Those who responded "cold" to the question "are your hands cold or warm?" and those who responded "cold" to the question "are your feet cold or warm?" were classified as the CHHF group. Those who responded "warm" or "normal" to the both of these questions were classified as the non-CHHF group. We excluded those who stated that they were cold in response to only 1 of these 2 questions because the presence of CHHF symptoms appeared to be unclear. For similar reasons, we excluded those who stated that they were unsure in response to either question.

2.3. Questionnaire on Digestion. The questionnaire included 9 items that refer to common complaints in Korea and were derived from descriptions in the Rome II classification [11]. In addition to these 9 items, the questionnaire included items on 3 topics that are needed to apply pattern identification in Korean medicine: digestion status ("how is your digestion?"), motion sickness, and exhaustion when hungry. The definition for each symptom was based on the description presented in Rome II [12]. The subjects were asked to answer the questionnaire based on their usual status within the past 6 months, which was chosen because the Glasgow Dyspepsia Severity Score [13] includes evaluations of symptoms during the latest 6 months and because the same symptom duration was presented in the most recent Rome III classification.

The details of each question were as follows. To the question "how is your digestion?" the subjects chose either "1. good" or "2. bad." To the question "how is your appetite?" and an item related to anorexia, the subjects chose "1. very good," "2. good," "3. average," or "4. not good." The criteria for these responses were as follows: "1. very good" refers to the desire to eat more foods despite satiety after meal; "2. good" refers to the case in which one feels hungry at mealtimes and wants to eat food; "3. average" refers to the case in which one eats meals at mealtimes but does not have a good appetite; and "4. not good" refers to the case in which one has no appetite at mealtimes and does not have a good sense of taste, even when eating. For the items on dyspepsia symptoms (discomfort in the upper abdomen, vomiting, motion sickness, exhaustion when hungry, belching, epigastric burning, postprandial fullness, nausea, epigastric pain, and abdominal bloating), respondents were asked to choose 1 of the following answers: "1. often," "2. sometimes," and "3. rarely." "1. often" refers to greater than or equal to 2 times per week, "2. sometimes" refers to greater than or equal to 3 times per month, and "3. rarely" refers to less than or equal to 2 times per month (Supplementary Table 1; see Supplementary Material available online at http://dx.doi.org/ $10.1155 / 2016 / 8948690)$.

2.4. Statistical Analysis. The statistical program SPSS 21.0 for Windows (IBM Corp., Armonk, NY, USA) was used for statistical analysis. The general characteristics of the subjects were matched using a propensity score consisting 


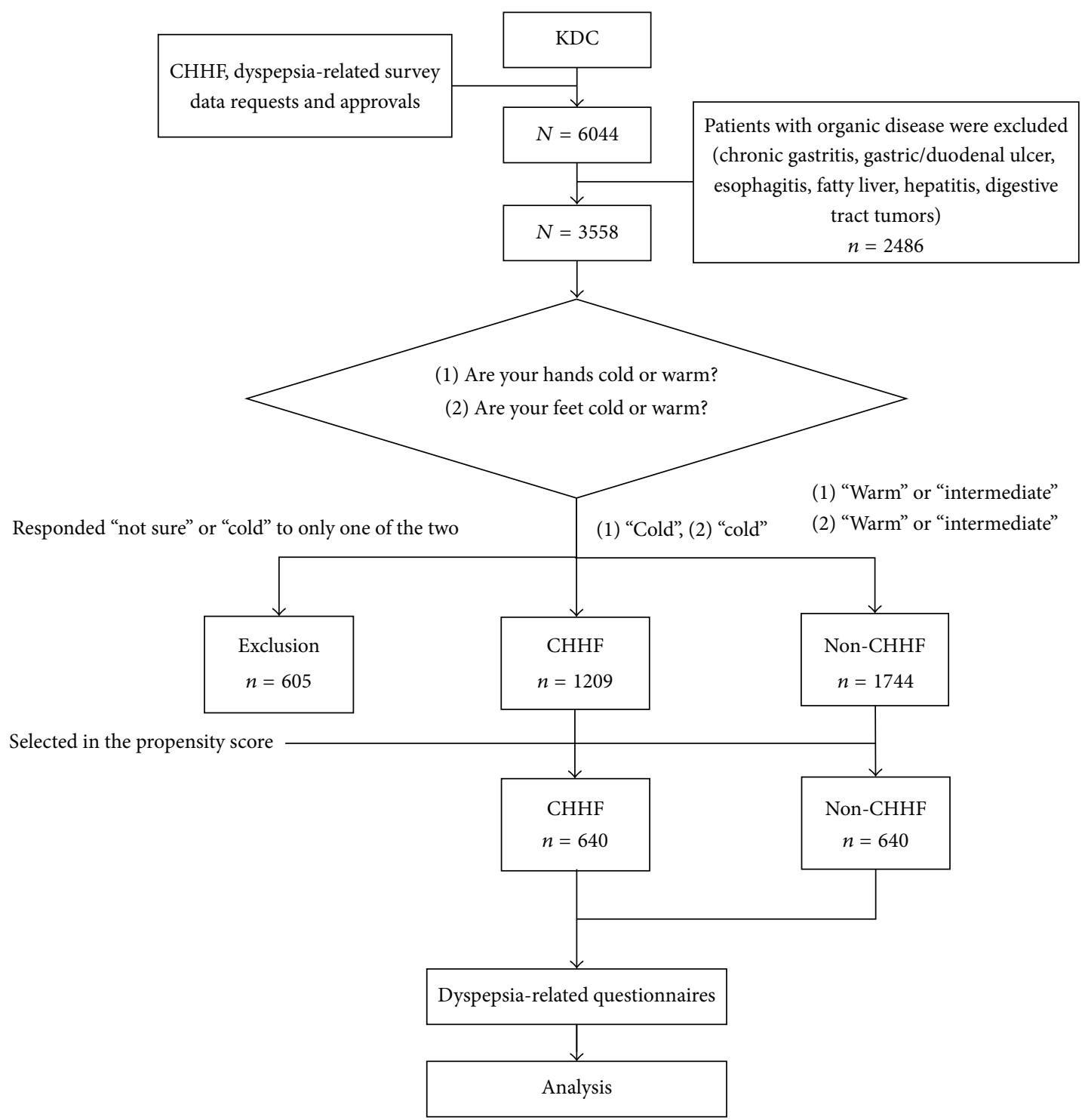

FIGURE 1: Flow chart of the study. KDC: Korean Medicine Data Center; CHHF: cold hypersensitivity in the hands and feet; non-CHHF: noncold hypersensitivity in the hands and feet.

of sex, age, and BMI, with the matching process involving a minimum distance scoring method. Figure 2 shows the alteration in propensity score distribution between the matched $\mathrm{CHHF}$ and non-CHHF groups. These physical characteristics were presented as frequencies and percentages or means \pm standard deviations. Between-group comparisons were performed using the chi-square test (for categorical variables) and the independent-samples $t$-test (for continuous variables). The chi-square test was used to analyze the frequencies and percentages of responses to digestion-related questions in the $\mathrm{CHHF}$ and non-CHHF groups. In addition, logistic regression was performed to calculate the odds ratios (ORs) for dyspepsia in the propensity-matched group as well as in the original groups. The OR was determined for each dyspepsia-related item in the CHHF group compared to the non-CHHF group. The statistical significance level was set at $P<0.05$.

\section{Results}

3.1. Demographic Characteristics. The number of subjects in the original $\mathrm{CHHF}$ and non-CHHF groups was 1209 and 1744 , respectively. The total study sample included more women $(n=1958 ; 66.3 \%)$ than men $(n=995 ; 33.7 \%)$. The female-to-male ratio was much higher in the $\mathrm{CHHF}$ group (983 women, $81.4 \%$, versus 226 men, 18.7\%) than in the total study sample. The mean ages in the CHHF and non-CHHF groups were 44.6 and 47.4 years, respectively. The mean height and weight in the non-CHHF group were $1.7 \mathrm{~cm}$ taller and $6.8 \mathrm{~kg}$ heavier, respectively, than the corresponding values in the CHHF group. The mean BMIs in the CHHF and non-CHHF groups were 22.0 and 24.1, respectively. The general characteristics were significantly different between $\mathrm{CHHF}$ and non-CHHF groups before matching (all $P<$ $0.001)$. After propensity score matching, the total number 


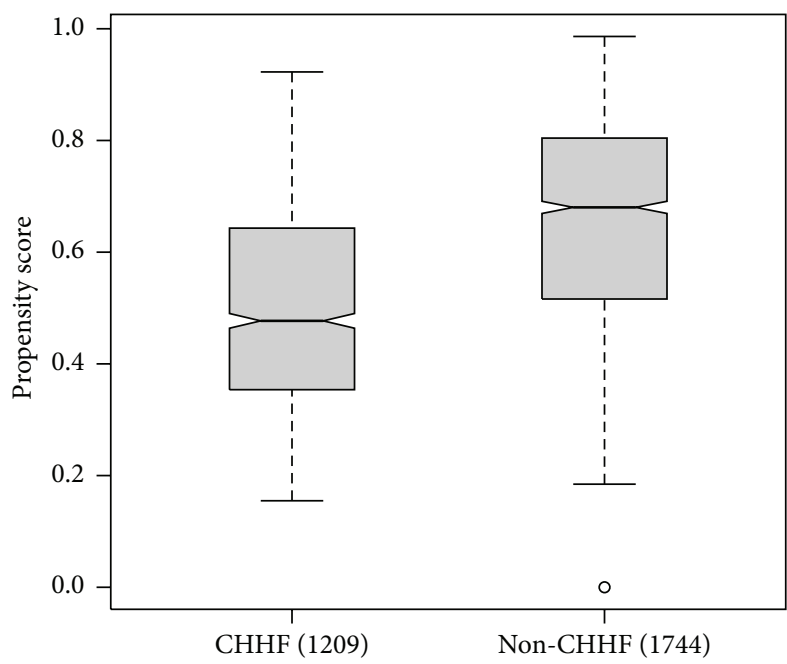

(a)

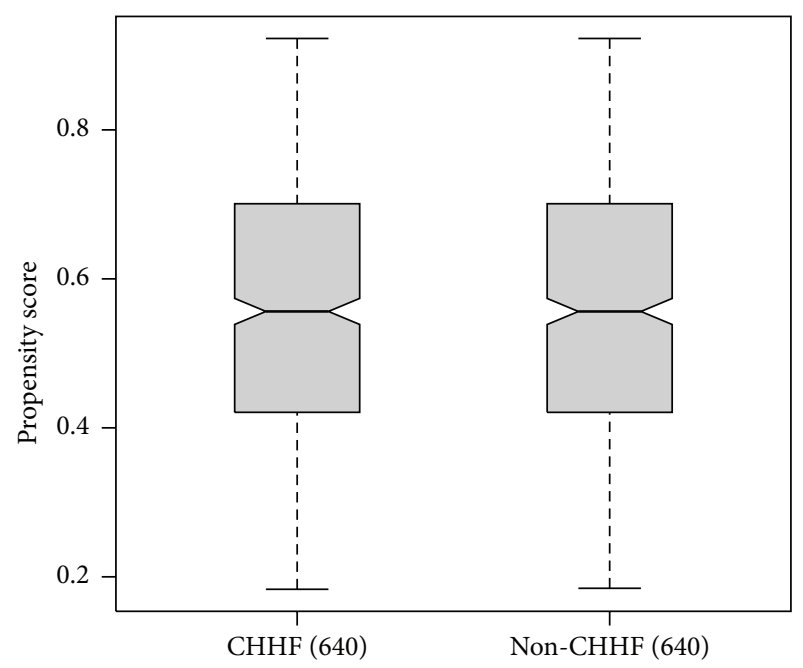

(b)

FIGURE 2: Comparison of the propensity score between the CHHF group and non-CHHF group before and after propensity matching. (a) Propensity score before matching; (b) propensity score after matching. CHHF: cold hypersensitivity in the hands and feet; non-CHHF: noncold hypersensitivity in the hands and feet.

TABLE 1: General characteristics of the study subjects.

\begin{tabular}{|c|c|c|c|c|c|c|}
\hline \multirow[b]{2}{*}{ Variable } & \multicolumn{3}{|c|}{ Before matching } & \multicolumn{3}{|c|}{ After matching } \\
\hline & $\begin{array}{c}\text { CHHF } \\
(n=1209)\end{array}$ & $\begin{array}{c}\text { Non-CHHF } \\
(n=1744)\end{array}$ & $P$ value & $\begin{array}{c}\text { CHHF } \\
(n=640)\end{array}$ & $\begin{array}{c}\text { Non-CHHF } \\
\quad(n=640)\end{array}$ & $P$ value \\
\hline \multicolumn{7}{|l|}{ Sex } \\
\hline Male & $226(18.7)$ & $769(44.1)$ & \multirow{2}{*}{$<0.001$} & $168(26.3)$ & $173(27)$ & \multirow{2}{*}{0.752} \\
\hline Female & $983(81.3)$ & $975(55.9)$ & & $472(73.8)$ & $467(73)$ & \\
\hline Age (y) & $44.6 \pm 13.8$ & $47.4 \pm 14.8$ & $<0.001$ & $44.9 \pm 14.8$ & $45 \pm 14.4$ & 0.858 \\
\hline Height $(\mathrm{cm})$ & $161.3 \pm 7.6$ & $163.1 \pm 8.8$ & $<0.001$ & $161.6 \pm 8.1$ & $161.2 \pm 8.0$ & 0.375 \\
\hline Weight (kg) & $57.4 \pm 8.8$ & $64.2 \pm 11.0$ & $<0.001$ & $59.8 \pm 8.8$ & $59.4 \pm 9.1$ & 0.447 \\
\hline BMI $\left(\mathrm{kg} / \mathrm{m}^{2}\right)$ & $22 \pm 2.8$ & $24.1 \pm 3.2$ & $<0.001$ & $22.9 \pm 2.8$ & $22.8 \pm 2.8$ & 0.731 \\
\hline
\end{tabular}

Results are presented as $n(\%)$ or mean \pm standard deviation.

CHHF: cold hypersensitivity in the hands and feet; Non-CHHF: noncold hypersensitivity in the hands and feet; BMI: body mass index.

of patients in each group was 640 , with no statistically significant differences in general characteristics between the groups (Table 1).

3.2. Chi-Square Tests of the Relationship between CHHF and Dyspepsia. Before matching, the CHHF group and nonCHHF group significantly differed $(P<0.001)$ in every dyspepsia item. A higher proportion of the CHHF group reported bad digestion and not good appetite compared to the non-CHHF group, and the frequency of all dyspepsia symptoms (discomfort in the upper abdomen, vomiting, motion sickness, exhaustion when hungry, belching, epigastric burning, postprandial fullness, nausea, epigastric pain, and bloating) was also higher.

After matching, significant differences were detected in digestion, postprandial fullness, bloating $(P<0.001)$, discomfort in the upper abdomen, motion sickness, epigastric burning, postprandial fullness, nausea, and appetite $(P<$ $0.05)$. The frequency of dyspepsia symptoms was higher in the CHHF group compared to the non-CHHF group, while there was no statistically significant difference between the two groups in items of vomiting, exhaustion when hungry, belching, and epigastric pain (related to digestion) (Table 2).

3.3. The Odds Ratios for Dyspepsia according to CHHF Status. As can be seen in Table 3 and Supplementary Figure 1, ORs were used to investigate the differences in dyspepsia between the CHHF and non-CHHF groups. Before propensity matching, a significant between-group difference was observed for all items.

After matching analyses, bad digestion, motion sickness, postprandial fullness, bloating $(P<0.001)$, vomiting, epigastric burning, nausea, and epigastric pain $(P<0.05)$ significantly differed between the groups, and there was no significant difference for not good appetite, discomfort in the upper abdomen, exhaustion when hungry, and belching. The OR was highest for bad digestion (2.423) before matching and for bloating after matching (1.883) (Table 3).

For the OR of each response ("often" and "sometimes") for dyspepsia symptoms after matching, both responses 
TABLE 2: Dyspepsia in the CHHF and non-CHHF groups before and after propensity matching.

\begin{tabular}{|c|c|c|c|c|c|c|}
\hline \multirow[b]{2}{*}{ Variable } & \multicolumn{3}{|c|}{ Before matching } & \multicolumn{3}{|c|}{ After matching } \\
\hline & $\begin{array}{l}\text { CHHF } \\
n(\%)\end{array}$ & $\begin{array}{c}\text { Non-CHHF } \\
n(\%)\end{array}$ & $P$ value & $\begin{array}{c}\text { CHHF } \\
n(\%)\end{array}$ & $\begin{array}{c}\text { Non-CHHF } \\
n(\%)\end{array}$ & $P$ value \\
\hline \multicolumn{7}{|l|}{ Digestion } \\
\hline Good & $814(67.3)$ & $1453(83.3)$ & \multirow{2}{*}{$<0.001$} & $440(68.8)$ & $513(80.2)$ & \multirow{2}{*}{$<0.001$} \\
\hline Bad & $395(32.7)$ & $291(16.7)$ & & $200(31.3)$ & $127(19.8)$ & \\
\hline \multicolumn{7}{|l|}{ Appetite } \\
\hline Extremely good & $74(6.1)$ & $131(7.5)$ & \multirow{4}{*}{$<0.001$} & $43(6.7)$ & $45(7.0)$ & \multirow{4}{*}{0.045} \\
\hline Good & $608(50.3)$ & $1014(58.1)$ & & $319(49.9)$ & $366(57.2)$ & \\
\hline Average & $423(35)$ & $508(29.1)$ & & $223(34.9)$ & $188(29.4)$ & \\
\hline Not good & $103(8.5)$ & $91(5.2)$ & & $54(8.5)$ & $41(6.4)$ & \\
\hline \multicolumn{7}{|c|}{ Discomfort in the upper abdomen } \\
\hline Often & $74(6.1)$ & $40(2.3)$ & \multirow{3}{*}{$<0.001$} & $32(5.0)$ & $17(2.7)$ & \multirow{3}{*}{0.038} \\
\hline Sometimes & $441(36.5)$ & $410(23.5)$ & & $210(32.8)$ & $193(30.2)$ & \\
\hline Rarely & $694(57.4)$ & $1294(74.2)$ & & $398(62.2)$ & $430(67.2)$ & \\
\hline \multicolumn{7}{|l|}{ Vomiting } \\
\hline Often & $5(0.4)$ & $5(0.3)$ & \multirow{3}{*}{$<0.001$} & $3(0.5)$ & $3(0.5)$ & \multirow{3}{*}{0.094} \\
\hline Sometimes & $133(11)$ & $98(5.6)$ & & $67(10.5)$ & $45(7.0)$ & \\
\hline Rarely & $1071(88.6)$ & $1641(94.1)$ & & $570(89.1)$ & $592(92.5)$ & \\
\hline \multicolumn{7}{|l|}{ Motion sickness } \\
\hline Often & $40(3.3)$ & $19(1.1)$ & \multirow{3}{*}{$<0.001$} & $16(2.5)$ & $11(1.7)$ & \multirow{3}{*}{0.001} \\
\hline Sometimes & $327(27)$ & $292(16.7)$ & & $165(25.8)$ & $113(17.7)$ & \\
\hline Rarely & $842(69.6)$ & $1433(82.2)$ & & $459(71.7)$ & $516(80.6)$ & \\
\hline \multicolumn{7}{|c|}{ Exhaustion when hungry } \\
\hline Often & $132(10.9)$ & $120(6.9)$ & \multirow{3}{*}{$<0.001$} & $60(9.4)$ & $41(6.4)$ & \multirow{3}{*}{0.057} \\
\hline Sometimes & $524(43.3)$ & $617(35.4)$ & & $260(40.6)$ & $245(38.3)$ & \\
\hline Rarely & $553(45.7)$ & $1007(57.7)$ & & $320(50)$ & $354(55.3)$ & \\
\hline Belching & & & & & & \\
\hline Often & $135(11.2)$ & $125(7.2)$ & & $70(10.9)$ & $53(8.3)$ & \\
\hline Sometimes & $408(33.7)$ & $578(33.1)$ & $<0.001$ & $217(33.9)$ & $224(35.0)$ & 0.272 \\
\hline Rarely & $666(55.1)$ & $1041(59.7)$ & & $353(55.2)$ & $363(56.7)$ & \\
\hline Epigastric burning & & & & & & \\
\hline Often & $48(4.0)$ & $41(2.4)$ & & $19(3.0)$ & $17(2.7)$ & \\
\hline Sometimes & $354(29.3)$ & $396(22.7)$ & $<0.001$ & $194(30.3)$ & $156(24.4)$ & 0.049 \\
\hline Rarely & $807(66.7)$ & $1307(74.9)$ & & $427(66.7)$ & $467(73.0)$ & \\
\hline Postprandial fullnes & & & & & & \\
\hline Often & $73(6.0)$ & 47 (2.7) & & $32(5.0)$ & $19(3.0)$ & \\
\hline Sometimes & $326(27.0)$ & $285(16.3)$ & $<0.001$ & $166(25.9)$ & $120(18.8)$ & 0.001 \\
\hline Rarely & $810(67.0)$ & $1412(81.0)$ & & $442(69.1)$ & $501(78.3)$ & \\
\hline Nausea & & & & & & \\
\hline Often & $32(3.6)$ & $20(1.6)$ & & $13(2.8)$ & $12(2.6)$ & \\
\hline Sometimes & $228(25.6)$ & $187(15.2)$ & $<0.001$ & $121(25.7)$ & $74(16.2)$ & 0.002 \\
\hline Rarely & $631(70.8)$ & $1024(83.2)$ & & $336(71.5)$ & $370(81.1)$ & \\
\hline Epigastric pain (rela & & & & & & \\
\hline Often & $40(3.3)$ & $22(1.3)$ & & $12(1.9)$ & $9(1.4)$ & \\
\hline Sometimes & $250(20.7)$ & $223(12.8)$ & $<0.001$ & $118(18.4)$ & $90(14.1)$ & 0.078 \\
\hline Rarely & $919(76.0)$ & $1499(86.0)$ & & $510(79.7)$ & $541(84.5)$ & \\
\hline Bloating & & & & & & \\
\hline Often & $76(6.3)$ & $51(2.9)$ & & $35(5.5)$ & $16(2.5)$ & \\
\hline Sometimes & $422(34.9)$ & $419(24)$ & $<0.001$ & $228(35.6)$ & $157(24.5)$ & $<0.001$ \\
\hline Rarely & $711(58.8)$ & $1274(73.1)$ & & $377(58.9)$ & $467(73.0)$ & \\
\hline
\end{tabular}

$P$ values are calculated from chi-square tests of the CHHF versus non-CHHF groups.

CHHF: cold hypersensitivity in the hands and feet; Non-CHHF: noncold hypersensitivity in the hands and feet.

Sample questions: digestion: "how is your digestion?"; appetite: "how is your appetite?"; symptoms (discomfort in the upper abdomen, vomiting, motion sickness, exhaustion when hungry, belching, epigastric burning, postprandial fullness, nausea, epigastric pain, and bloating): "do you have any of the following symptoms?" 
TABLE 3: The odds ratios and 95\% confidence intervals for dyspepsia before and after propensity matching according to CHHF status.

\begin{tabular}{|c|c|c|c|c|c|}
\hline \multirow[b]{2}{*}{ Variable } & \multirow[b]{2}{*}{ Non-CHHF } & \multicolumn{2}{|c|}{ Before matching } & \multicolumn{2}{|c|}{ After matching } \\
\hline & & $\begin{array}{c}\text { CHHF } \\
\text { OR }(95 \% \mathrm{CI})\end{array}$ & $P$ value & $\begin{array}{c}\text { CHHF } \\
\text { OR (95\% CI) }\end{array}$ & $P$ value \\
\hline Digestion: bad & Ref & $2.423(2.036-2.884)$ & $<0.001$ & $1.836(1.421-2.372)$ & $<0.001$ \\
\hline Appetite: not good & Ref & $1.693(1.264-2.268)$ & $<0.001$ & $1.349(0.885-2.056)$ & 0.165 \\
\hline Discomfort in the upper abdomen & Ref & $2.134(1.825-2.495)$ & $<0.001$ & $1.245(0.990-1.566)$ & 0.061 \\
\hline Vomiting & Ref & $2.053(1.572-2.680)$ & $<0.001$ & $1.515(1.031-2.226)$ & 0.035 \\
\hline Motion sickness & Ref & $2.008(1.689-2.389)$ & $<0.001$ & $1.641(1.264-2.130)$ & $<0.001$ \\
\hline Exhaustion when hungry & Ref & $1.621(1.398-1.879)$ & $<0.001$ & $1.238(0.994-1.542)$ & 0.057 \\
\hline Belching & Ref & $1.207(1.041-1.400)$ & 0.013 & $1.065(0.854-1.329)$ & 0.573 \\
\hline Epigastric burning & Ref & $1.490(1.268-1.751)$ & $<0.001$ & $1.347(1.060-1.711)$ & 0.015 \\
\hline Postprandial fullness & Ref & $2.095(1.769-2.481)$ & $<0.001$ & $1.615(1.255-2.077)$ & $<0.001$ \\
\hline Nausea & Ref & $2.038(1.656-2.509)$ & $<0.001$ & $1.716(1.260-2.336)$ & 0.001 \\
\hline Epigastric pain & Ref & $1.931(1.598-2.332)$ & $<0.001$ & $1.393(1.044-1.858)$ & 0.024 \\
\hline Bloating & Ref & $1.899(1.625-2.219)$ & $<0.001$ & $1.883(1.489-2.382)$ & $<0.001$ \\
\hline
\end{tabular}

CHHF: cold hypersensitivity in the hands and feet; Non-CHHF: noncold hypersensitivity in the hands and feet; OR: odds ratio; CI: confidence interval; Ref: reference.

"Non-CHHF" was employed as the reference in every analysis.

Sample questions: digestion: "how is your digestion?"; appetite: "how is your appetite?"; symptoms (discomfort in the upper abdomen, vomiting, motion sickness, exhaustion when hungry, belching, epigastric burning, postprandial fullness, nausea, epigastric pain, and bloating): "do you have any of the following symptoms?" Symptoms are the sum of "often" and "sometimes" responses.

("often" and "sometimes") for bloating significantly differed between groups. There was a significant difference in either "often" or "sometimes" responses for discomfort in the upper abdomen, vomiting, motion sickness, exhaustion when hungry, epigastric burning, postprandial fullness, nausea, and epigastric pain, and there was no difference in belching (Supplementary Figure 1).

\section{Discussion}

We chose to investigate the relationship between $\mathrm{CHHF}$ (including various cold symptoms) and indigestion because of the "spleen and 4 extremities" theory that was described in Huangdi's classic text [2], which is one of the most famous works in oriental medicine. As stated in this theory, the spleen is included in the human digestive system and controls the passage of nutrition to the 4 limbs, and therefore symptoms in the 4 extremities are thought to relate to the function of the spleen. This theory is taken into consideration when prescribing acupuncture or herbal medicine in the clinic. Furthermore, because the prevalence of functional dyspepsia is high $(8-30 \%)$, we expected that it would be relatively easy to investigate correlations between $\mathrm{CHHF}$ and dyspepsia [7].

$\mathrm{CHHF}$ refers to a condition in which one experiences discomfort in daily living because of cold symptoms in the limbs. It is more inclusive than Raynaud's phenomenon and includes decreased temperature in the hands and feet, as well as the subjective sensation of cold. CHHF is suspected to induce spastic peripheral vasoconstriction, but no specific, certain cause has yet been identified. In Korea, CHHF is a relatively common symptom and occurs more often in women than in men [5]. The diagnosis and treatment of $\mathrm{CHHF}$ are a relatively active topic of research in Korea, including recent studies by Park et al. [14] and Hur et al. [5]. In another study of the relationship between CHHF and diseases, Tokunaga et al. [15] investigated "Hie," which refers to oversensitivity to coldness. Kondo and Okamura [6] also investigated the relationship between $\mathrm{CHHF}$ and the Cornell Medical Index (CMI).

Dyspepsia is a digestive function disorder that refers to the collective symptoms of the upper gastrointestinal tract. In general, the term "dyspepsia" denotes functional dyspepsia; in this study, we therefore excluded persons who had been diagnosed with chronic gastritis, gastroduodenal ulcers, esophagitis, fatty liver, hepatitis, and digestive tract tumors. Through these exclusions, we sought to remove as many cases of organic dyspepsia as was feasible. In Rome III [16], functional dyspepsia is defined as the presence of symptoms including postprandial satiety, early satiety, gastric pain, and epigastric burning without any organic disease. Although the Rome III definition is generally accepted, the diagnostic standard of Rome III has not been applied strictly to many cases in clinical practice, and therefore the diagnostic period of dyspepsia has remained somewhat controversial [17].

In the present study, the data were compiled from questionnaire responses that had been collected by KDC. Participants who noted having cold hands and feet were assigned to the CHHF group, while participants who had neither cold hands nor cold feet were assigned to the non-CHHF group. Between-group differences in indigestion were analyzed using the KDC digestion questionnaire. Several recent studies have used the KDC data, including those by Do et al. [18] study on the Sasang constitutional diagnostic method, Chae et al. [19] on the development of the Sasang constitution questionnaire, and Jang et al. [20] on metabolic syndrome. 
The aim of this study was to verify whether there was a difference in functional dyspepsia frequency according to the presence of $\mathrm{CHHF}$ and, if so, which of the various digestionrelated symptoms differed. In summary, patients with $\mathrm{CHHF}$ showed a high frequency of dyspepsia, with this tendency generally maintained after matching. Bloating in particular significantly differed between groups both before and after matching: the highest OR after matching was 1.883 and the responses "often" and "sometimes" were significantly more common in the CHHF group.

The general characteristics of subjects differed markedly depending on the presence of CHHF. In the CHHF group, the female-to-male ratio (81\%) was much higher compared to the non-CHHF group (56\%), and the mean age and BMI were higher in the non-CHHF group $\left(47.4\right.$ years and $24 \mathrm{~kg} / \mathrm{m}^{2}$ versus 44.6 years and $22 \mathrm{~kg} / \mathrm{m}^{2}$, resp.). We concluded that these differences could harbor considerable bias in examining the relation between $\mathrm{CHHF}$ and dyspepsia. Therefore, the patients were matched in the CHHF group and non-CHHF group using the propensity score matching method (640 patients per matched group). There was no difference in sex, BMI, and age after matching. These differences in general characteristics confirmed that CHHF was influenced by sex and BMI, as in previous studies $[5,6]$.

This study suggests that there is a correlation between $\mathrm{CHHF}$ and functional dyspepsia. In the chi-squared test shown in Table 2, the CHHF group and non-CHHF group significantly differed in all items before matching and in digestion, appetite, discomfort in the upper abdomen, motion sickness, epigastric burning, postprandial fullness, nausea, and bloating after matching. There was no significant difference in vomiting, exhaustion when hungry, belching, or epigastric pain.

As shown in Table 3, the OR for the development of dyspepsia symptoms (bad digestion, not good appetite, and sum of "often" and "sometimes" for the occurrence of each symptom) was increased in every item before matching in the CHHF group, and a significantly increased OR was observed for bad digestion, vomiting, motion sickness, epigastric burning, postprandial fullness, nausea, epigastric pain, and bloating after matching. A significant OR was not observed for not good appetite, discomfort in the upper abdomen, exhaustion when hungry, or belching. These results revealed a slight difference in the items with significant differences according to the analysis method but generally supported a high frequency of dyspepsia in the CHHF group.

In this study, we observed a significant difference between the frequencies of indigestion in participants who did and did not have CHHF. Previously, Tokunaga et al. [15] found that symptom frequencies differed according to the presence of Hie (oversensitivity to coldness), and Nietert et al. [21] found that Raynaud's phenomenon was associated with undiagnosed vascular disease. Together with these earlier investigations, the present study provides evidence supporting the notion that the human disease state of "cold" can endanger human health.

However, this study has several limitations. First, it relied on a cross-sectional design and used qualitative and subjective indicators. Second, the KDC survey was provided to patients who had been admitted to a group of traditional Korean medicine clinics and hospitals in Korea, rather than to members of the general population. Third, survey respondents were assigned to the $\mathrm{CHHF}$ and non-CHHF groups based on the questionnaire answers, rather than a doctor's diagnostic findings. Fourth, although the questions on dyspepsia were for the most part based on the functional dyspepsia symptoms described in Rome II [12], a verified questionnaire on dyspepsia was not used. Additionally, the questions referring to digestion status were 2-point scales, questions referring to appetite were 4-point scales, and dyspepsia symptoms were 3-point scales. Therefore we could not sum the total scores for examination. Fifth, we also used self-reported survey responses to determine which patients had organic digestive diseases (and subsequently exclude the identified patients from our analysis). However, the presence or absence of organic disease was not verified based on doctors' examinations, such as endoscopic findings.

Therefore, we believe that a follow-up study is necessary to accurately define the relationship between CHHF and functional dyspepsia. Additional studies to delineate the mechanism between dyspepsia and CHHF are also needed. We hope that future studies will better reveal the precise correlation between these two symptoms and their underlying cause.

\section{Conclusions}

In this study, we were able to verify that patients with $\mathrm{CHHF}$ have more chronic (lasting more than 6 months) functional dyspepsia symptoms, especially bloating.

\section{Conflict of Interests}

The authors declare that they have no competing interests.

\section{Authors' Contribution}

Kwang-Ho Bae conceived the study design and drafted the paper. Ki-Hyun Park and Jong-Hyang Yoo collected and analyzed the questionnaire data. Ju Ah Lee, Youngseop Lee, and Siwoo Lee helped with the previous study and reviewed the paper. All of the authors contributed critically to the final paper and approved the final version. Youngseop Lee and Siwoo Lee are equal contributors.

\section{Acknowledgments}

This research was supported by the Bio \& Medical Technology Development Program of the National Research Foundation (NRF), which is funded by the Ministry of Science; ICT \& Future Planning (NRF-2014M3A9D7034335); and the research program of the Korea Institute of Oriental Medicine (K15101).

\section{References}

[1] H. J. Kim, H. S. Bae, S. U. Park, S. K. Moon, J. M. Park, and W. S. Jung, "Clinical approach to the standardization of oriental medical diagnostic pattern identification in stroke patients," 
Evidence-Based Complementary and Alternative Medicine, vol. 2011, Article ID 768492, 7 pages, 2011.

[2] World Health Organization, WHO International Standard Terminologies on Traditional Medicine in the Western Pacific Region, World Health Organization, Geneva, Switzerland, 2007.

[3] H. Ryu, H. Lee, H. Kim, and J. Kim, "Reliability and validity of a cold-heat pattern questionnaire for traditional Chinese medicine," Journal of Alternative and Complementary Medicine, vol. 16, no. 6, pp. 663-667, 2010.

[4] J. Y. Song, M. J. Kim, W. S. Sung et al., "Efficacy and safety of herb medication according to cold-heat tendency of knee osteoarthritis patients," The Journal of Korean Acupuncture \& Moxibustion Medicine Society, vol. 29, no. 5, pp. 97-108, 2012.

[5] Y.-M. Hur, J.-H. Chae, K. W. Chung et al., "Feeling of cold hands and feet is a highly heritable phenotype," Twin Research and Human Genetics, vol. 15, no. 2, pp. 166-169, 2012.

[6] M. Kondo and Y. Okamura, "Cold constitution: analysis of the questionnaire," Nihon Sanka Fujinka Gakkai Zasshi, vol. 39, no. 11, pp. 2000-2004, 1987.

[7] U. C. Ghoshal, R. Singh, F.-Y. Chang, X. Hou, B. C. Y. Wong, and U. Kachintorn, "Epidemiology of uninvestigated and functional dyspepsia in Asia: facts and fiction," Journal of Neurogastroenterology and Motility, vol. 17, no. 3, pp. 235-244, 2011.

[8] S.-Y. Choi, M.-J. Lee, E.-K. Kim et al., "A study on the correlation of cold hypersensitivity and common health indexes in young women," Korean Journal of Obstetrics \& Gynecology, vol. 24, no. 4, pp. 62-70, 2011.

[9] E.-Y. Nam, J.-I. Lee, G.-M. Kim, and H.-J. Kim, "A study on the correlation of cold hypersensitivity and digestive function," Journal of Oriental Obstetrics and Gynecology, vol. 27, no. 1, pp. 140-151, 2014.

[10] H. J. Jin, Y. Baek, H. S. Kim, J. Ryu, and S. Lee, "Constitutional multicenter bank linked to Sasang constitutional phenotypic data," BMC Complementary and Alternative Medicine, vol. 15, article 46, 2015.

[11] C. Huh, C. H. Yang, J. G. Jang et al., "Functional dyspepsia and subgroups in Korea and short term outcome of therapeutic trial of cisapride; Multicenter study," Journal of Neurogastroenterology and Motility, vol. 4, no. 1, pp. 1-12, 1998.

[12] D. A. Drossman, "The functional gastrointestinal disorders and the Rome II process," Gut, vol. 45, no. 2, pp. II1-II5, 1999.

[13] E. M. el-Omar, S. Banerjee, A. Wirz, and K. E. McColl, "The Glasgow Dyspepsia Severity Score-a tool for the global measurement of dyspepsia," European Journal of Gastroenterology \& Hepatology, vol. 8, no. 10, pp. 967-971, 1996.

[14] K.-S. Park, K.-I. Park, J.-W. Kim et al., "Efficacy and safety of Korean red ginseng for cold hypersensitivity in the hands and feet: a randomized, double-blind, placebo-controlled trial," Journal of Ethnopharmacology, vol. 158, part A, pp. 25-32, 2014.

[15] H. Tokunaga, K. Munakata, K. Katayama et al., "Clinical data mining related to the Japanese kampo concept 'hie' (oversensitivity to coldness) in men and pre- and postmenopausal women," Evidence-Based Complementary and Alternative Medicine, vol. 2014, Article ID 832824, 9 pages, 2014.

[16] D. A. Drossman, "The functional gastrointestinal disorders and the Rome III process," Gastroenterology, vol. 130, no. 5, pp. 13771390, 2006.

[17] N. Manabe, K. Haruma, J. Hata et al., "Clinical characteristics of Japanese dyspeptic patients: is the Rome III classification applicable?" Scandinavian Journal of Gastroenterology, vol. 45, no. 5, pp. 567-572, 2010.
[18] J.-H. Do, E. Jang, B. Ku, J.-S. Jang, H. Kim, and J. Y. Kim, “Development of an integrated Sasang constitution diagnosis method using face, body shape, voice, and questionnaire information," BMC Complementary and Alternative Medicine, vol. 12, article 85, 2012.

[19] H. Chae, S. Lee, S. H. Park, E. Jang, and S. J. Lee, "Development and validation of a personality assessment instrument for traditional Korean medicine: sasang personality questionnaire," Evidence-Based Complementary and Alternative Medicine, vol. 2012, Article ID 657013, 12 pages, 2012.

[20] E. Jang, Y. Baek, K. Park, and S. Lee, “The sasang constitution as an independent risk factor for metabolic syndrome: propensity matching analysis," Evidence-Based Complementary and Alternative Medicine, vol. 2013, Article ID 492941, 6 pages, 2013.

[21] P. J. Nietert, S. R. Shaftman, R. M. Silver et al., "Raynaud phenomenon and mortality: 20+ years of follow-up of the Charleston Heart Study cohort," Clinical Epidemiology, vol. 2015, article 7, pp. 161-168, 2015. 


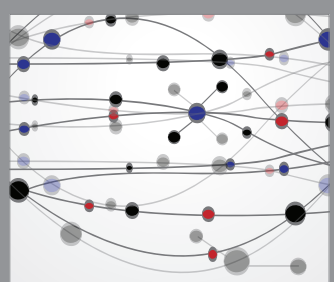

The Scientific World Journal
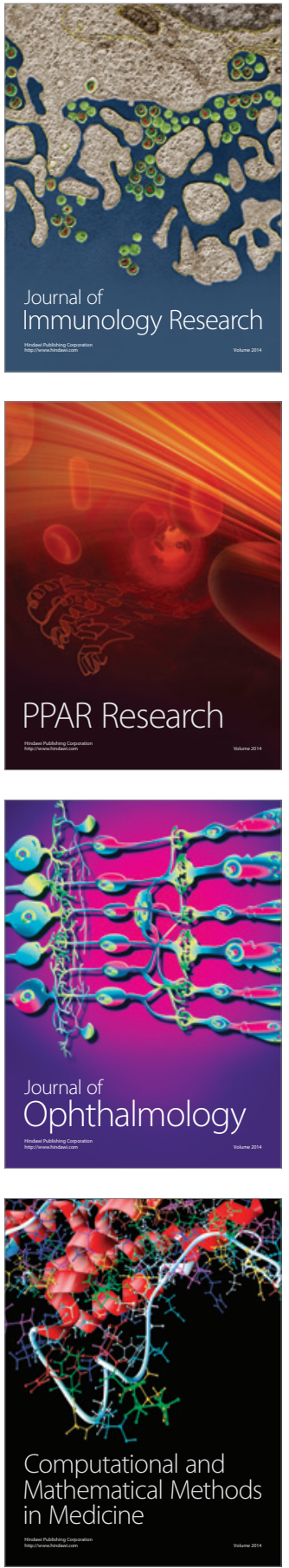

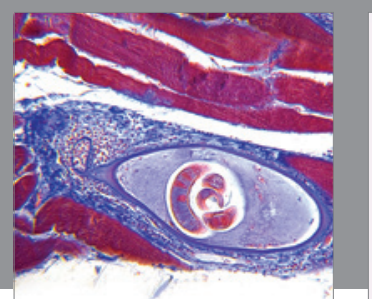

Gastroenterology Research and Practice

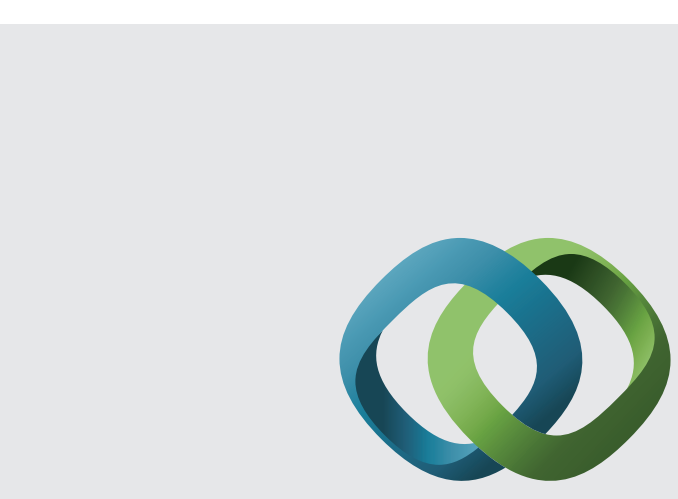

\section{Hindawi}

Submit your manuscripts at

http://www.hindawi.com
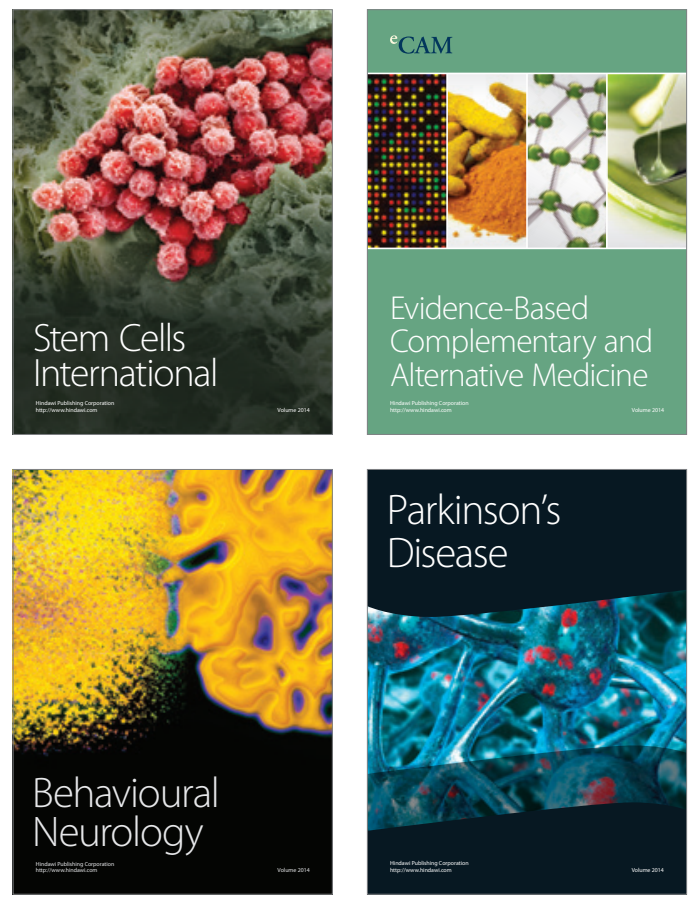
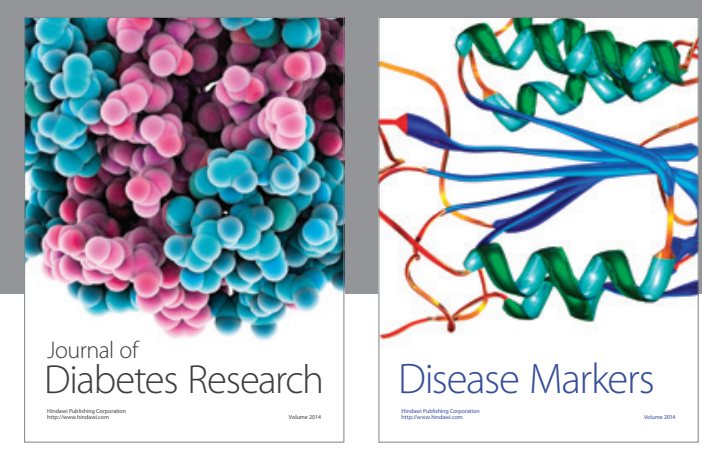

Disease Markers
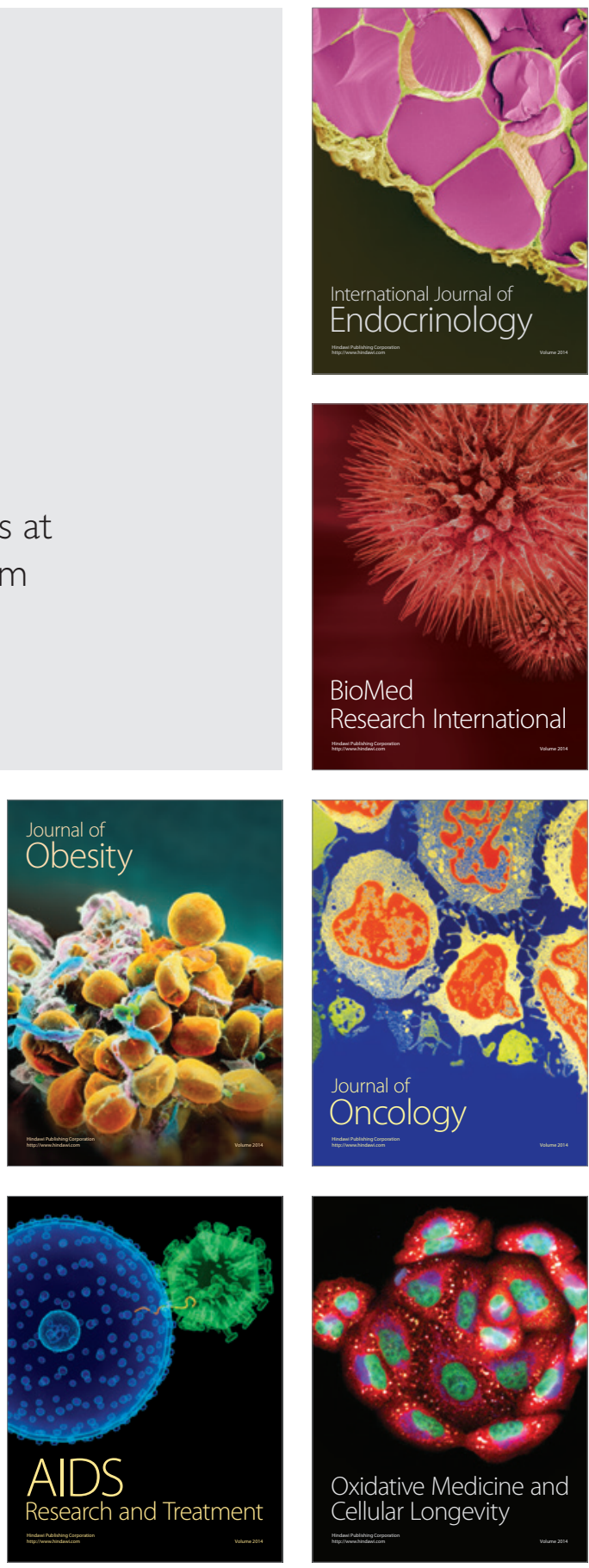